\title{
Les défis de l’approvisionnement alimentaire : acteurs, lieux et liens
}

\author{
Pierre Janin
}

À paraître dans Janin P. et Fofiri Nzossié E.-J. (coord.), 2019. Approvisionnement alimentaire : entre ressources et pouvoirs, Revue internationale des études du développement, $\mathrm{n}^{\circ} 237$, mars 2019, Editions de la Sorbonne, Paris.

\begin{abstract}
Résumé :
Dans un monde d'instabilités et d'inégalités croissantes, l'approvisionnement alimentaire apparaît comme un défi permanent pour les gouvernants et pour les sociétés. Au-delà de ses modalités pratiques d'organisation, il renvoie à deux enjeux fondamentaux : celui de la maîtrise stratégique des flux et celui des liens géographiques et sociaux au sein des systèmes d'approvisionnement. Cet article introductif insiste sur les complémentarités des approches couramment mobilisées pour aborder cette question, récemment peu étudiée. En associant de manière étroite le champ des ressources et des pouvoirs, il rappelle combien la fourniture de denrées n'est pas seulement une question technique de prix, de volumes et de flux, mais dépend in fine des relations établies entre différents types d'acteurs : états, entreprises, collectivités, réseaux marchands... On constate que dans certains contextes autoritaires (Pays du Golfe, Amérique latine), la présence de l'État-organisateur reste incontournable, tandis que, hors crise (Afrique sub-saharienne), une plus large place est laissée à des dynamiques plus informelles.
\end{abstract}

Mots-clés: approvisionnement; système alimentaire; production agricole; commercialisation; politique d'importation ; État ; sécurité alimentaire

Dans le domaine alimentaire, l'approvisionnement apparaît comme un défi récurrent à relever, pour tous les acteurs concernés (États, entreprises, réseaux marchands, familles,...) : il est, non seulement, complexe à organiser, mais également difficile à maitriser (Rapoport, 1993), a fortiori dans les environnements instables et peu sécurisés des pays en développement. Les problèmes d'approvisionnement concernent aussi bien les villes que les campagnes ${ }^{1}$, même si ces dernières semblent encore priorisées en termes d'intervention. Au demeurant, l'approvisionnement pose deux enjeux fondamentaux : celui de la maîtrise stratégique des flux, celui des liens géographiques et sociaux (de la " connexion " pourraiton dire), que nous allons nous efforcer de préciser et d'éclairer dans cette introduction.

Mettant aux prises des acteurs, des ressources et des lieux, l'approvisionnement est toujours très lié à un contexte. Le temps et l'espace y constituent des marqueurs essentiels : ils se matérialisent par des discontinuités, des accélérations, lorsque les ressources se raréfient ou que les échanges se contractent ou se réorientent; ils expriment des régularités lorsque l'approvisionnement est, tant bien que mal, assuré et que les variables prix et qualité n'aggravent pas l'inaccessibilité. Au-delà de l'expression d'une offre et d'une demande, d'une relation entre besoins et capacités, l'approvisionnement est une question politique, marquée par des arrangements, des alliances et des concurrences, qui participent de cette distorsion. Liant l'économique au politique, parfois avec une relecture des héritages historiques, les analyses proposées, dans ce dossier, montrent le caractère complexe de cette question, comme les difficultés à assurer durablement un approvisionnement adéquat.

\footnotetext{
${ }^{1}$ En milieu rural, si les difficultés d’approvisionnement paraissent plutôt prévisibles et cycliques, elles sont plus aléatoires et fractionnées en ville.
} 


\section{Des systèmes complexes d'approvisionnement : produits et acteurs écartelés entre valeur et identité}

L'approvisionnement est transversal et touche à la fois à la production agricole, au commerce des denrées et à leur redistribution sociale et géographique. Ce qui contribue à rendre difficile la stabilisation du champ d'analyse². II renvoie implicitement à l'idée que l'on chercherait à l'assurer pour le plus grand nombre : ce qui n'est pas toujours le cas. D'un point de vue conceptuel, c'est autant la question de la transmission du risque (de pénurie, d'inaccessibilité,...) que de la mutualisation - ou pas - des ressources qui est posée ${ }^{3}$. En complément, du point de vue des "mangeurs", l'approvisionnement peut être analysé en termes de droits et d'obligations individuels ou collectifs (perte ou mobilisation pour les faire valoir, responsabilité vis-à-vis des dépendants alimentaires ou des vulnérables). S'approvisionner, c'est aussi faire des choix (Padilla, 1997 : p. 24). Ils peuvent être contraints, donnant même parfois corps à l'idée de dilemmes (Rittel and Webber, 1973 ; Hattemer et Sierra, 2012), mais également dépendre de préférences (Bonnecase, 2016) qui viennent orienter les pratiques d'achat, de préparation et de consommation. Qui plus est, en temps ordinaire, l'approvisionnement alimentaire peut également être abordé en termes de distinction sociale par les denrées achetées et consommées (Chevalier, 2015) comme de satisfaction (Héron, 2016).

Les dynamiques d'approvisionnement dépendent de nombreuses variables, telles que la disponibilité saisonnière des produits, le volume des denrées en circulation, les niveaux de prix de gros ou de détail, la distance géographique et l'état des infrastructures routières,... Toutes s'enchevêtrent et exercent une influence positive ou négative sur l'approvisionnement effectif, en temps et en heure, a fortiori dans les contextes très insécurisés, en cas de conflit, lorsque les infrastructures sont déficientes ou en cas d'État absentéiste ou failli. Par ailleurs, l'approvisionnement se pose en des termes très différents, voire contradictoires du point de vue du fournisseur, du transporteur, du régulateur ou du mangeur.... Pour les experts et pour les décideurs, l'approvisionnement joue un rôle clé pour " atteindre la sécurité alimentaire et nutritionnelle" (Reinert, 2015). L'approvisionnement constitue aussi un vecteur d'influence et de changement : pour des aliments mondialisés et standardisés (pizza, burger, chawarma, tacos,...) et pour la promotion de denrées de terroir (Petrini, 2006 ; Ostrom, 2009 ; CAAAQ, 2008 ; BertonOfouémé, 2017). Quant aux réseaux marchands qui l'organisent, ils sont presque toujours, à la fois, réactifs et adaptatifs.

Du local au global, différents types de canaux peuvent être identifiés : privé, associatif, familial, institutionnel (Bertrand, 1991 ; Wade et Lançon, 2015) ; marchands et non marchands, entre cellules familiales distantes ou proches (Tawodzera, 2013), ou via la prise en charge de dépendants alimentaires (Frayne, 2010). Ils renvoient à autant de niveaux de stratification : informelle de rue, semi-informelle des boutiquiers, traditionnelle des marchés ouverts, couverte des supérettes et autres vastes malls, sur fond $d^{\prime}$ 'individuation des pratiques et de segmentation des pouvoirs d'achat. On observe ainsi un rapide rattrapage en Afrique subsaharienne ( $d$ 'abord anglophone) par rapport aux situations urbaines en Amérique Latine. Ils peuvent être assignés socialement comme du point de vue du genre (Allen and Sachs, 2009) : femmes, mères et épouses sont ainsi très présentes dans l'approvisionnement domestique (comme dans la petite transformation), tandis que le commerce régional et lointain est plutôt masculin.

Au cœur de l'approvisionnement, temporalités et spatialités sont indissociables (Karg and al., 2016) : les réseaux marchands disposent souvent de leur propre réseau d'information et de collecte de denrées tandis qu'à l'opposé, les citadins diversifient les rythmes et les modes d'approvisionnement. Les études réalisées dans le champ de l'approvisionnement l'ont été, à différentes échelles, souvent à partir d'approches combinant la géographie et l'économie.

\footnotetext{
${ }^{2}$ La question de l'approvisionnement renvoie également à celle de sécurisation alimentaire, dans la mesure où elle privilégie "l'analyse des processus permettant de gérer durablement les approvisionnements et d'en assurer la sécurité d'une façon jugée fiable par différentes parties prenantes » (Hubert, 2006).

3 Dans l'absolu, le champ d'analyse va au-delà, avec les interrogations sur la durabilité des modèles de production et de consommation alimentaires, compte tenu de la raréfaction et de la fragilisation des facteurs de ressources.
} 
La première approche s'intéresse aux " échanges villes-campagnes ", à l'échelle locale ou régionale (Franqueville, 1997 ; Calas, 1999), avec des difficultés concrètes pour les mesurer: depuis plus d'une décennie, la problématique des circuits courts de proximité est venue renouveler cette approche, tandis que l'approche territoriale, associant évolutions des systèmes productifs, des modèles de consommation et des dynamiques d'extraversion s'impose, conduisant, par exemple, à un phénomène de diversification à l'échelle ouest-africaine (OCDE, 2013 ; Bricas et al., 2016).

La seconde approche s'appuie, depuis les années 1980, sur la notion de filière ou de circuit (Hugon, 1997 ; Padilla et Bencharif, 2001) insistant sur les goulets d'étranglement à lever (Bilinsky and Swindale, 2005) que l'analyse actuelle, en termes de chaînes de valeur alimentaire - et de partage de la valeur entre les acteurs - précise, tout en s'ouvrant à des dimensions nouvelles de justice et d'équité qui viennent enrichir et bousculer les termes de la sécurisation alimentaire. Plusieurs pistes se dessinent pour améliorer les marges des différents acteurs : la valorisation patrimoniale (et donc politique) de certaines denrées, emblématiques d'un terroir et d'une culture alimentaire, comme le relève Paula Vasquez Lezama, avec les galettes de maïs, dans son article sur la situation actuelle du Venezuala ou encore les actions menées en termes de labellisation dans le champ du commerce équitable (dossier à suivre dans la Revue ${ }^{4}$ ). Dans leur article sur les filières de feuilles de manioc à Kinshasa, Papy Bonkena Bokombola et Patience Mpanzu Balomba insistent, pour leur part, sur le rôle de la transformation agro-alimentaire - dans ses procédés comme dans ses structures de vente - afin de favoriser leur acheminement distant ou leur montée en gamme; toutefois, ici encore, les producteurs familiaux ne captent qu'une part réduite de leur valeur monétaire.

Dans les deux approches, l'approvisionnement est plutôt abordé d'un point de vue socio-technique, avec des étapes, des flux, des produits. À cet effet, nombre de travaux se sont efforcés de décrire les étapes de mise en marché des denrées agricoles (Festas, 2006 ; Fofiri Nzossié, 2013), la fonctionnalité et les embarras du transport (Padilla et Bencharif, 2001) ou encore évolution de l'offre de détail en milieu urbain (Bricas et al., 2004). D'autres, souvent des économistes, ont cherché à spatialiser les écarts de prix (Araujo-Bonjean et al. 2010 ; OCDE, 2013) et à retracer les co-évolutions futures de l'offre, de la demande, des flux d'échanges (prospective Dualine du Cirad et de l'INRA au début des années 2010).

Au-delà, c'est bien la "qualité » de la chaîne, du producteur au consommateur final (agri-food supply chain management ou supply chain security $)^{5}$ dont il est question. Si les termes " chaîne d'approvisionnement " et " chaîne de valeur " sont souvent mobilisés et utilisés de manière, peu ou prou, interchangeables, des distinctions existent : la première pense efficacité de la logistique pour satisfaire des besoins essentiels (PAM, 2013), la seconde renvoie à la répartition inégale des marges entre acteurs des filières et pose donc le débat du point de vue de la gouvernance des filières. Au-delà de la nécessité de " fournir à tout prix ", le champ s'est enrichi également des enjeux de qualité sanitaire et nutritionnelle des denrées mises en marché 6 - dans et hors crise.

Actuellement, c'est désormais cette thématique qui s'impose dans ce champ, même si la pluralité des régimes de gouvernance (Moustier and Renting, 2015 ; Cossi Hinnou et al., 2016 ; Amanor, 2009) est encore peu évoquée, en dépit de leur stabilisation, depuis le milieu des années 1980 par H. Friedmann, si ce n'est au sein des mouvements favorables à une réinscription politique des rapports marchands. Ainsi, un décalage important existe entre ceux qui parlent de gouvernance démocratique et de justice

\footnotetext{
${ }^{4}$ Pour certains, l'obtention d'un label d'appellation d'origine et une bio-certification, passant par une action concertée associant l'Etat, les acteurs socio-économiques et les consommateurs, sont en cours ou envisagées (attiéké pour la Côte d'Ivoire).

${ }^{5}$ Ce type de recherche occupe désormais une place de plus en plus importante (Luo and al. 2018). Pour une définition de "food supply chain ", lire Marsden and al., 2000.

${ }^{6}$ Ce prisme a émergé plus tardivement en Afrique subsaharienne que dans les autres aires géographiques (Delisle, 1998) : la crise nigérienne de 2005 est ainsi fondatrice pour ce qui est des produits thérapeutiques de traitement de la malnutrition sévère, tandis que les exigences des mangeurs citadins se construisent au fur et à mesure de la médiatisation des produits mondialisés et du renforcement de l'offre commerciale des supermarchés.
} 
alimentaire (associations, organisations paysannes, syndicats,...) et ceux qui se bornent à l'évoquer d'un point de vue technique (management des risques) ${ }^{7}$. Cette sociologie politique de l'approvisionnement présente l'avantage d'aborder les rapports de pouvoir entre les différents types d'acteurs impliqués (Porter and al., 2007) qui restent liés à la trajectoire historique des systèmes socio-spatiaux considérés (Ariyo and al., 2001 ; Guyer, 1987).

Ainsi, jusqu'à une période récente, peu de travaux ayant une approche intégrée pouvaient être mobilisés. Seuls ceux réalisés, à la fin des années 1990, par O. Argenti, M. Azagrande et M. Padilla (repris par Armandariz and al., 2016) pour les villes d'Afrique subsaharienne, autour des "systèmes d'approvisionnement et de distribution alimentaires " (SADA) ou " food supply and distribution system " (FSDS), s'en réclamaient. Très précis d'un point de vue méthodologique, ils avaient vocation à orienter la réflexion pour l'action publique en prenant appui sur la pluralité d'échelles et d'objectifs.

Plus fondamentalement, l'approvisionnement alimentaire s'adosse à la notion de système alimentaire (Labonne, 1986; Rastoin et Ghersi, 2010) dont elle constitue, à la fois, la fonction centrale et la formalisation concrète. Dans le même ordre d'idée, des scientifiques poussent, plus loin encore, l'allégorie entre dynamique instable d'approvisionnement et métabolisme du corps vivant pour en restituer toute la complexité (Bognon, 2014). Dans les faits, en matière d'approvisionnement, les réalités sont nettement plus prosaïques : il s'agit plus de répondre à des besoins quotidiens que repenser des stratégies globales d'approvisionnement, du local à l'international (AFD, 2015).

À bien des égards, la réémergence du thème de la sécurisation des approvisionnements puise à différentes prises de conscience, tant technocratique que citoyenne : celle des effets d'une mondialisation malheureuse, favorable à la spécialisation agricole sur la base d'avantages comparatifs pas toujours réels ; celle des dégâts effectifs des modèles agricoles productionnistes sur la biodiversité et sur la santé ; et celle de l'intrication inexorable de crise environnementale, agricole et alimentaire aux conséquences cumulatives et durables. Entre incertaine soutenabilité, perte de confiance et menaces, ce sont les capacités des systèmes d'approvisionnement (encore moins résilients) qui sont questionnées, et même souvent, mises en doute (Pouch, 2009). Elles reposent, avec acuité, à différentes échelles, la question du rôle de l'État, de ses missions en matière d'approvisionnement comme de sa capacité à organiser le champ, en période de tension comme en dehors des situations de crise avec des groupes d'acteurs hétérogènes: humanitaires et développeurs, privés, sociaux intermédiaires (syndicats, associations, réseaux sociaux, etc.).

En parallèle, la problématique de l'approvisionnement se reconfigure peu à peu autour/avec de nouveaux engagements, martelés par l'agenda des ODD : par exemple, le souci de durabilité conduit, peu à peu, à favoriser l'émergence de circuits courts et la reterritorialisation de la production en ville ${ }^{8}$ ou à leur proximité (Parfitt and al., 2010); celui d'équité incite, pour sa part, à la valorisation patrimoniale des produits locaux ou/et au rééquilibrage du partage de la valeur (FAO 2018); quant à celui d'autonomie, il plaide pour une réduction de la dépendance ancienne (Coussy, 1990) des systèmes alimentaires aux échanges extérieurs (aide, importations,...). D'où, y compris dans des pays peu concernés par les problèmes d'approvisionnement, des réflexions sur la réorganisation des systèmes et des logiques d'approvisionnement (food supply chains) (Hinrichs, 2013).

Fort de ce qui précède, ce dossier fait le choix de privilégier les échelles méso- et macro- d'analyse des défis de l'approvisionnement. Il accorde aussi une large place à l'action publique qui en traite, impliquant souvent une pluralité d'acteurs (privés, associatifs,...). En revanche, il laisse de côté l'échelle domestique - dans et hors marché - des approvisionnements, qui renvoie plutôt aux différentes pratiques alimentaires d'achat, de préparation et de consommation, comme de l'autoproduction (food selfprovisioning) ayant fait l'objet de nombreuses études (notamment suivi de panels de ménages dans le cadre d'enquêtes locales ou nationales).

\footnotetext{
${ }^{7}$ La restructuration des rapports de pouvoir, au cœur des systèmes d'approvisionnement, constitue à la fois un champ d'analyse porteur et une nouvelle voie pour l'action publique ou citoyenne.

8 Participant à la conquête d'une d'autonomie alimentaire tout en luttant contre les pertes et les gaspillages.
} 


\section{Sécuriser les approvisionnements : le contrôle des flux plutôt que leur localisation}

Sécuriser les approvisionnements en denrées constitue un dilemme permanent, quel que soit l'échelle de décision, le régime politique et le niveau de développement ${ }^{9}$. Deux voies existent. La première consiste à créer les conditions durables d'une production diversifiée de proximité : elle a été testée au cours des décennies passées avec des politiques nationales d'autosuffisance et elle revient en force dans le cadre des transitions agricoles et alimentaires. La seconde, longuement analysée dans ce dossier, consiste à mettre en place un contrôle des flux (avec plus d'efficacité que celui des prix), quelles que soient leur origine et leur distance, quitte à s'affranchir des conditions agro-écologiques et sociétales de production. Elle a été clairement privilégiée à l'échelle mondiale, depuis la période coloniale, renforcée par les politiques favorables au marché (du riz ou du blé par exemple), jusqu'à une période très récente.

Le caractère stratégique de l'approvisionnement n'est plus à débattre, lorsque que sont concernées des populations pauvres et vulnérables frappées par des pénuries importantes ${ }^{10}$ : il relève d'urgences à satisfaire qui ne souffrent pas réellement de controverses. Dans ce type de situation, les besoins en matière d'approvisionnement conduisent presque inévitablement à des «actions prioritaires immédiates ", quitte à établir des " règles d'exception " selon le principe afférent (Agamben), surtout dans les régimes autoritaires, qui craignent les interpellations médiatiques et les mobilisations sociales (Engels, 2013). Dans la plupart des cas, les discussions sur les modalités de prise en charge ${ }^{11}$ interviendront, une fois la crise passée, quitte à faire évoluer les cadres normalisés d'intervention.

La fourniture de denrées est alors d'autant plus essentielle que leur acheminement reste souvent incertain, par manque de moyens, du fait de lenteurs ou de l'éloignement de certaines populations rurales déplacées. Elle l'est plus encore dans les zones de conflit endémique (Nord-est du Nigeria, Est de la RDC, Somalie, Nord de I'Irak,...) où les denrées produites ou reçues via l'aide humanitaire, sont susceptibles d'être accaparées, détournées et revendues par des groupes militarisés. Dans ce type de situation, le contrôle violent de l'approvisionnement alimentaire renvoie à des rapports de domination et imprime ses marques coercitives sur les corps, au sens foucaldien du terme (Nally, 2011).

Hors contexte de crise, dans de nombreux pays en développement, l'approvisionnement alimentaire est encore considéré comme une des missions régaliennes et souveraines ${ }^{12}$ de l'État (Siobhan and Swensson, 2017). Ce dernier est, " tel un bon père de famille ", soit chargé de créer les conditions nécessaires pour que le marché puisse continuer à remplir son mandat d'approvisionnement ${ }^{13}$, soit sommé d'assurer un niveau minimal de sécurité en s'y substituant le cas échéant, sans toujours y parvenir (Mander, 2015) ${ }^{14}$, mais ayant toujours à cœur de définir une vision stratégique à moyen terme (Rastoin, 2015).

L’histoire récente fournit des exemples très contrastés de ce qu'organiser l'approvisionnement veut dire pour un État. Prenons l'exemple de l'Inde qui dispose d'un système public visant à garantir des prix

\footnotetext{
${ }^{9}$ En septembre 2018, le gouvernement britannique vient ainsi de nommer un sous-secrétaire d'Etat à l'approvisionnement en prenant conscience que son autosuffisance ( $25 \%$ pour les fruits et les légumes) était loin d'être assurée avec un Brexit imminent.

${ }^{10}$ Cela l'est plus encore dans les situations de conflit où la survie immédiate des enfants est menacée et lève tout débat inutile.

${ }^{11}$ Faut-il plutôt distribuer des denrées, des bons ou de l'argent ? Est-il réaliste de prétendre contrôler les prix de détail sans système coercitif ? Est-il éthique de demander une contrepartie ?

${ }^{12}$ Le terme est pris ici dans son sens commun, au croisement de l'idée d'indépendance et d'autosuffisance alimentaire (Pouch, 2011), et non pas en référence à l'ensemble des mouvements sociaux et des mobilisations transnationales (Hrabanski, 2011) se réclamant de l'objectif politique de "souveraineté alimentaire ", en termes d'accès à une nourriture suffisante et de qualité, comme par la maitrise et le contrôle des processus et des facteurs de production agricole (Mormont, 2007).

${ }^{13}$ Ce rôle central de régulateur est ainsi clairement au cœur de l'émergence des « trajectoires agro-alimentaires de produits nutritionnels de qualité » pour les vulnérables (Maestre and al., 2017).

${ }^{14}$ Cette prise de responsabilité est non seulement espérée mais revendiquée dans le cadre de mobilisations sociales populaires, en cas de pénurie (Hossain and Kalita, 2009).
} 
mimima d'achat aux producteurs, à constituer des stocks et à les redistribuer aux consommateurs les plus pauvres (Lutringer, 2018) : il conduit progressivement " à une concentration des ressources et donc un ciblage indirect à la fois sur certaines régions et sur certaines catégories de producteurs » contrevenant quelque peu à sa mission de péréquation. Pour sa part, la Chine constitue un cas ambivalent de réussite en matière d'approvisionnement : grâce à une vigoureuse politique agricole ${ }^{15}$, elle dispose de stocks stratégiques (blé, maïs, soja, sucre et riz) mobilisables en cas de récoltes insuffisantes mais, dans le même temps, lutte pour réduire une dépendance croissante aux importations alimentaires (Pouch, 2017) qui s'est traduite par des investissements massifs dans des terres cultivables à l'étranger (Chaumet, 2015) et orienter vers son marché les ressources produites, quitte à risquer d'amplifier les tensions géopolitiques (Mottet, 2016).

En régime autoritaire, la tentation est forte pour un appareil d'État de rester au cœur de l'approvisionnement alimentaire, soit parce qu'il politise fortement la quête nationale de sécurité alimentaire, soit qu'il parce qu'il gère de manière patrimoniale et redistributive l'accès aux ressources. Le document de Paula Vasquez Lezama illustre pleinement cette tension entre ressources et pouvoirs. Partant de la situation alimentaire dramatique des Vénézuéliens, elle montre combien cette pénurie, loin d'être conjoncturelle, s'inscrit dans un ensemble de dysfonctionnements où se mêlent subventions coûteuse aux denrées de première nécessité, via la reconversion de la rente pétrolière, et pratiques corruptives au sein des réseaux de distribution gérés par le gouvernement. Depuis 2013 , la dégradation de la situation financière du pays, a conduit à une politisation renforcée des distributions et, au final, de la pénurie dont les effets s'expriment désormais par des flux massifs d'émigrants.

Les monarchies pétrolières des pays du Golfe, étudiées par Mathieu Brun et par Damien Calais, constituent deux exemples intéressants en termes de politique nationale volontariste d'approvisionnement. Structurellement déficitaires en céréales produites localement, compte tenu de facteurs de production défavorables, elles ont mis en place des politiques agricoles volontaristes mais très coûteuses et des circuits locaux de distribution de produits à prix modéré, sans parvenir à réduire le caractère extraverti de leur approvisionnement. En parallèle, les gouvernants ont d'abord réalisé des investissements fonciers importants à l'étranger tout en organisant une intégration verticale de l'approvisionnement via des entreprises. Ici, sans doute plus qu'ailleurs, du fait de la centralité du pouvoir, les jeux d'alliance diplomatique et clientéliste jouent un rôle important dans la stratégie mise en œuvre. Les politiques étatiques d'approvisionnement contribuent donc de manière évidente à conforter le pouvoir politique au sens où l'entendent D. Bourmaud et D. Darbon (1990).

A contrario, dans de nombreux autres contextes, même si l'approvisionnement est perçu comme stratégique par l'État, celui n'a pas ou plus les moyens d'intervenir : les politiques d'ajustement structurel sont passées par là. La fourniture de produits alimentaires a été déléguée à des acteurs marchands (oligopole importateur et agro-transformateur, lorsque le tissu entrepreneurial est suffisamment développé), depuis les marchés agricoles internationaux ${ }^{16}$ en passant par les marchés nationaux et locaux. Jusqu'à la crise alimentaire de 2007-2008, ces marchés constituaient une offre crédible, pour assurer compléter un bilan alimentaire déficitaire, laissant de côté l'éventuel risque de flambée des prix.

Très vite, les gouvernants ont alors mis en place des politiques ciblées en matière de sécurisation des approvisionnements : ambitieux programmes de relance hydro-agricole, acquisitions de terres agricoles dans certaines zones peu peuplées, négociation de contrats d'approvisionnement de gré à gré avec certains pays producteurs ou groupes agro-alimentaires...). Le bilan dressé, pour quelques pays

\footnotetext{
${ }^{15}$ Soutien à la recherche agronomique, accroissement des subventions à la production, stabilisation des prix du marché intérieur, investissement massif dans la construction d'infrastructures rurales et d'irrigation.

${ }^{16}$ Cette circulation mondiale des denrées de base s'explique, pour une large part, par les choix hérités de la période coloniale, en matière de spécialisation sur la base d'avantages comparatifs.
} 
africains ${ }^{17}$, montre que l'on oscille entre des plaidoyers volontaristes, pas toujours suivis d'effet, et des opérations plus médiatiques destinées à valoriser des demi-succès. Mais, dans l'ensemble, ces actions n'ont pas réellement infléchi les trajectoires d'approvisionnement: certaines sont restées limitées, d'autres se sont avérées coûteuses, au risque d'en revenir à l'option la plus évidente du recours au marché (illustrant ainsi la place de l'entrepreneurial food regime).

Depuis les choses ont avancé : les options techniques de régulation des marchés - pour assurer des approvisionnements de qualité à des prix stabilisés - sont clairement identifiées (Galtier et Vindel, 2012), mais buttent sur la manque d'engagement politique (Touzard et Temple, 2012). Une convergence de vues existe, désormais, sur la nécessité qu'il y à renforcer le rôle régulateur de l'Etat : elles s'inscrivent dans un nouveau cycle qui tend à le réhabiliter (Grégoire, Kobiané et Lange, 2018). Sont réapparues, çà et là, dans les pays d'Afrique subsaharienne notamment, des initiatives publiques, appuyées par les bailleurs tels que l'Union européenne et l'Agence française de développement et les institutions sousrégionales (le Comité inter-États de lutte contre la sécheresse au Sahel - CILSS -, l'Union économique et monétaire ouest-africaine - UEMOA) visant à reconstituer de stocks céréaliers de réserve, de l'espace régional au local, et, en milieu urbain, à créer de filets sociaux de base (Caisses de résilience de la FAO, par exemple, depuis 2016) pour assurer une capacité minimale d'approvisionnement aux populations pauvres ${ }^{18}$.

Au cœur des crises également, cette volonté étatique peut s'exprimer: soit par un contrôle dans l'affectation sociale, ethno-communautaire et géographique des ressources de l'aide humanitaire, comme en Ethiopie ou au Zimbabwe, soit par la mise en place de dispositifs propres, via des programmes (banques de céréales, coupons alimentaires) et des sociétés de distribution alimentaire, dont la mission principale est d'assurer aux populations urbaines la disponibilité, la régularité et l'accessibilité des produits de consommation de base ${ }^{19}$.

Faute de quoi, les difficultés pour s'approvisionner perdureront pour ces populations, quand elles ne seront pas renforcées par les dynamiques de changement à l'œuvre dans un contexte de changement climatique accéléré : notamment celle de la marchandisation croissante des facteurs de ressources (eau, terres, plantes) et des inégalités croissantes qui pourront en découler, conduisant à l'aggravation des tensions alimentaires entre régions comme entre catégories de population. Par ailleurs, faute de vraies politiques publiques d'approvisionnement pour les villes - en dehors de subventions aux denrées de base - la question de l'insécurité alimentaire des citadins reste posée avec beaucoup d'acuité, compte tenu de leurs ressources monétaires variables, du fait de marchés de l'emploi exigus et peu formalisés. Une des voies envisagées actuellement consiste à re-territorialiser les politiques et les productions, via de nouvelles relations entre mondes ruraux et urbains.

\footnotetext{
${ }^{17}$ En 2008, on a relevé la Grande offensive pour l'alimentation et l'abondance (GOANA) au Sénégal, l'Initiative riz au Mali ou encore l'Offensive régionale pour la production alimentaire et contre la faim du CILSS qui évoquent toutes une action rapide, planifiée et efficace.

${ }^{18}$ Cette action s'inspire des programmes de cash transfers, de food vouchers et de food for work, délivrant des ressources de manière graduelle et répétée dans le temps, auprès des plus pauvres, en milieu rural comme urbain, depuis 2007-2008, dans plusieurs pays: Productive safety net programme (PNSP) en Ethiopie, Oportunidades à Mexico ou le National rural employment guarantee scheme (NREGS) en Inde. Ils permettent d'assurer une capacité minimale d'auto-approvisionnement familial sans en garantir toutefois la pérennité. Le programme éthiopien lie, de manière étroite et positive, sécurité alimentaire, nutrition et sécurisation foncière (Lavers, 2013).

${ }^{19}$ Exemple du réseau de banques de céréales au Mali en 2005 (Arditi, Janin et Marie, 2011) ou de la Mission de régularisation et d'approvisionnement des produits de base - MIRAP- au Cameroun créée en 2011.
} 


\section{Au cœur de l'approvisionnement : la problématique du lien (géographique et social) et des pouvoirs (marchands et politiques)}

Les notions de relation et de circulation sont au cœur de la question de l'approvisionnement ${ }^{20}$ : elles s'organisent autour d'une diversité de produits, de territoires et de lieux centraux, de règles et de normes, assurant une fonctionnalité aux systèmes d'échanges. Au-delà, c'est bien la nature du lien géographique et social, liant territoires et acteurs, qui est posée. Du coup, également celle de distance. Pour leur part, les transferts entre régions productrices et consommatrices, les péréquations entre zones excédentaires et déficitaires au sein de filières, tantôt courtes (Goossens et al., 1994), tantôt à plus longue distance, et plus ou moins institutionnalisées (Fromageot, 2005), sont toujours moins bien connus et référencés, compte tenu des difficultés pour les mesurer. En dépit de cela, ces échelles locales et régionales « comptent » pour beaucoup dans les dynamiques d'approvisionnement.

Ainsi, dans de nombreux pays, la connexion "rural-urbain » des systèmes d'approvisionnement est historiquement et sociologiquement forte, même si nombre de gouvernants ont reproduit un biais urbain via des cheap food policy, généralement défavorable aux petits producteurs familiaux ${ }^{21}$. Dès la fin des années 1980, l'amélioration du fonctionnement des marchés nationaux était une priorité (au Mali avec l'opération PRMC) de certains programmes d'ajustement structurel (Coussy, 1990): la promotion de filières locales en était une des résultantes, puisque l'on pariait sur un effet d'entrainement de la demande des consommateurs urbains sur les systèmes de production environnants (Temple et al. 2009), même si, de facto, l'appétence des « mangeurs » pour les produits alimentaires importés réduisait, peu à peu, la part des céréales locales consommés. Dans l'ensemble, cette connexion a bien fonctionné, comme le prouve l'absence de ruptures d'approvisionnement en céréales en Afrique subsaharienne pénuries céréalières en milieu urbain : les agricultures vivrières et familiales et les réseaux marchands ont su répondre à une hausse continuelle de la demande (Moustier, 1999 ; Acloque Desmulier, Gasselin, Rouillé d'Orfeuil, 2014), y compris dans des contextes où les infrastructures de transport, de contrôle sanitaire et de commerce sont déficientes ou peu formalisées (Chaléard et al. 2002). De même, une part non négligeable de l'approvisionnement des villes est également assurée par les agricultures urbaines et périurbaines (Bricas et al., 2004), souvent minutieuses, aux fonctions plurielles (économique, alimentaire, patrimoniale,...), mais soumises de plus en plus à de fortes concurrences d'usage (Abo-ElWafa and al., 2017).

Les relations en matière d'approvisionnement ne sont pas à sens unique : à certaines périodes, villes et campagnes sont même en concurrence pour accéder aux denrées. On a ainsi pendant longtemps sousestimé, à tort, les besoins marchands des producteurs ruraux. Or, ceux-ci ne sont pas uniquement liés à des événements brutaux conjoncturels, du fait de calamités naturelles réduisant le volume des récoltes, mais cycliques. Inversement, avec le phénomène de diversification alimentaire croissante (blé, riz et tubercules par exemple), dans les villes d'Afrique subsaharienne, l'intensité des échanges à l'échelle sous-régionale se renforce. Par ailleurs, au cœur des villes, l'accroissement des inégalités alimentaires d'accès contribue à structurer une diversité de marchés d'approvisionnement, tant informels que formels (Crush and Frayne, 2011) : si les petits marchés sont fréquentés par les classes populaires, les supermarchés s'adressent plutôt aux classes moyennes émergentes.

Pour leur part, dans ce numéro, Renato S. Maluf et Lidiane Fernandes Da Luz s'intéressent aux reconfigurations récentes des relations entre production, distribution et consommation au Brésil. À cet effet, ils mobilisent le concept " d'espace politique d'approvisionnement » impliquant une pluralité d'acteurs et de systèmes alimentaires. La participation sociale et la valorisation des cultures locales sont autant de leviers pour favoriser la promotion de l'accès aux aliments de qualité. Au demeurant, ces dynamiques semblent de nature à apporter des réponses aux attentes des mangeurs en termes de qualité, mais

\footnotetext{
${ }^{20}$ Certains parleront aussi de « système agri-urbain » (Robineau et Soulard, 2017).

${ }^{21}$ La question d'un rééquilibrage, passant par de vigoureuses politiques incitatives d'appui (Elbehri and al., 2013) est, certes, dans les esprits mais reste encore en devenir.
} 
surtout de proximité dans l'un des pays d'Amérique latine où la distance géographique et sociale peut renforcer certaines formes de marginalité (alimentaire).

Mieux, la dimension locale de l'approvisionnement apparaît comme une tendance de fond. Après une période de bas coûts consécutifs à la crise mondiale de 2007-2008, les prix énergétiques n'ont cessé de grimper, réévaluant sensiblement les coûts du transport à longue distance. Ceci plaide participent au redimensionnement géographique des systèmes alimentaires : le concept « d'espace de proximité " a le vent en poupe, prolongeant la notion de " bassin alimentaire " ou foodshed (Kloppenburg, Hendrickson and Stevenson, 1996). Avec lui, ce sont les relations entre producteurs et consommateurs qui sont rediscutées (par les scientifiques) et peu à peu reconfigurées (par certaines dynamiques communautaires).

Les actions visant à renforcer les circuits courts, de proximité, alternatifs, localisés se multiplient sur tous les continents (Chiffoleau, 2008 ; Deverre and Lamine, 2010 ; Van der Ploeg et al., 2012 ; Watts and al., 2005). Par ailleurs, les producteurs sont de plus en plus incités, par les institutions de développement, à s'impliquer dans les organisations censées les représenter et à répondre collectivement à des appels d'offres visant à approvisionner les marchés locaux. En parallèle, cette évolution s'est traduite par la montée en puissance de l'urban food planning ou des city region food systems (CRFS ${ }^{22}$ ) avec des choix forts en matière d'avitaillement/ravitaillement territorialisé de la part des institutions locales et régionales concernées (Pothukuchi and Kaufman, 1999). Dès le début de la décennie 2000, la FAO a mis en place son programme Food into cities $^{23}$, avant d'inscrire, depuis 2010 , dans ses plans d'action " des aliments pour les villes » comme priorité. Ceci constitue une nouveauté car les défis de l'approvisionnement étaient surtout posés pour les contextes ruraux.

Dans son article sur l'approvisionnement de La Havane, Riadh Mestiri montre comment une action politique de grande ampleur conduit à la construction progressive d'un système agraire urbain, en réponse à une situation d'isolement géo-économique très handicapante. Mais, l'objectif stratégique d'assurer la couverture des besoins alimentaires de la population de la capitale reste difficile à atteindre, en dépit d'un engagement étatique appuyé : d'abord par une phase de redistribution des terres et de coopérativisme et, plus récemment, par l'innovation agronomique des organopónicos et l'ouverture conjointe de marchés de proximité.

De tels exemples de reconfiguration des systèmes alimentaires autour d'une assise territoriale de proximité sont désormais nombreux, de l'Europe à l'Amérique Latine : ils sont l'expression d'une convergence forte entre mouvements favorables à la reconnaissance de certaines formes contractuelles d'agriculture paysanne et biologique, à la souveraineté alimentaire et à l'économie sociale et solidaire. II est évident que ce basculement ne se fera pas sans une implication forte des pouvoirs publics (via la mise en place de mécanismes juridiques), à l'instar du Programme d'acquisition des aliments ${ }^{24}$, par les structures associatives et publiques communales (restaurants populaires, écoles, crèches, prisons), au Brésil (Moruzzi Marques et Le Moal, 2015) qui favorise les petits producteurs locaux.

Outre les notions de relation et de circulation, le deuxième élément clé pour relire les systèmes d'approvisionnement est celui des logiques et des pratiques des acteurs impliqués (Hatcheu Tchawe, 2003), dessinant autant de rapports de pouvoir. Au cœur des connexions entre systèmes de production et de distribution, entre espaces ruraux et marchés urbains se dessinent de multiples formes de relation, d'alliance, de dépendance où d'arrangement (Robineau, 2013): entre producteurs/organisations paysannes et commerçants, entre élites marchandes et politiques, entre acteurs humanitaires et publics.

\footnotetext{
22 Un " système alimentaire ville-région » peut être défini comme " un ensemble complexe de réseaux d'acteurs, de processus et de relations ayant trait à la production alimentaire, la transformation, la commercialisation, le marketing et la consommation au sein d'une région géographique donnée ayant, plus ou moins, un centre urbain concentré, des périphéries urbaines et un hinterland rural ; un ensemble régional au sein duquel des flux de personnes, de biens et de services écosystémiques sont gérés » (Blay-Palmer et al., 2018, p. 3, traduction de l'auteur).

${ }^{23} \mathrm{http}: / /$ www.fao.org/fcit/food-marketing/fr/ (consulté en novembre 2018).

24 Il constituait une des composantes du programme Faim zéro mis en place par le gouvernement Lula à partir de 2003.
} 
Quel que soit le niveau de développement, de sécurité ou de stabilité d'une zone donnée, l'approvisionnement se décline autour d'un ensemble d'activités nécessitant des compétences techniques, un bon capital relationnel et une adaptabilité importante.

Quant aux normes et aux règlements, ils ne sont pas à négliger. S'ils jouent un rôle marquant en période de crise, allant presque jusqu'à régenter les interventions des acteurs étatiques et humanitaires ${ }^{25}$ (Enten, 2017), les choses paraissent moins contraignantes du point de vue des réseaux marchands locaux et régionaux : ces derniers bénéficient souvent de conditions plus souples - dont ils choisissent même parfois de s'affranchir - du fait de relations privilégiées avec l'appareil étatique ou d'une position oligopolistique (Poussart-Vanier, 2006). Dans les faits, ce sont eux qui assurent bien souvent la logistique de redistribution de l'aide ${ }^{26}$. Le bilan des promesses non tenues, pendant la crise alimentaire de 20072008, du point de vue des prix de détail, pour des denrées importées ayant bénéficié d'exonération de taxes à l'importation, est peu flatteur pour ces derniers.

Enfin, cette question des pouvoirs marchands est au cœur de l'analyse de Samuel Pinaud des dynamiques observées dans un espace sahélo-soudanien dans l'Est au Burkina Faso. Cette « distribution du pouvoir de marché » est étudiée, à partir d'une enquête de terrain auprès de commerçants céréaliers, à trois niveaux : celui de la structure des échanges, celui de l'organisation sociale du commerce et celui des stratégies mises en œuvre. Ce pouvoir de marché ne se limite pas à un avantage informationnel mais bénéficie aussi de la saisonnalité des disponibilités, des transferts et des spéculations. Plus dommageable pour les petits producteurs familiaux concernés, si la concurrence entre commerçants n'est pas absente, elle est socialement régulée, notamment en termes de prix. On se situe donc loin du cadre souhaité par les politiques favorables à l'amélioration du fonctionnement du marché.

En guise de conclusion, on pourrait insister sur deux points peu abordés dans ce dossier. Les évolutions des modes de production, de transformation et de consommation alimentaires vont se faire, à des rythmes différents, avec, à terme, un pouvoir accru de décision de la part du mangeur-acteur, au prix de luttes importantes avec les autres pouvoirs constitués. En contrepartie, et avec lui, viendront s'exprimer des aspirations multiples, parfois difficiles à satisfaire, susceptibles de créer de l'instabilité ou de la contradiction : entre qualité nutritionnelle, accessibilité économique, empreinte environnementale et valorisation sociétale, quelles priorités l'emporteront ? On pressent déjà également que des actions (et des politiques) plus efficientes, plus durables et mieux acceptées, passeront sans aucun doute par une meilleure contextualisation et territorialisation. Avec elles s'ouvre donc tout un champ de réflexion sur les manières de faire interagir (et non plus les opposer) des systèmes alimentaires pluriels pour une meilleure sécurisation.

\section{Pierre Janin}

Pierre Janin, docteur (Habilité à diriger des recherches - HDR) en géographie, est chercheur à l'Institut de recherche pour le développement (IRD). Il a dirigé l'UMR Développement et Sociétés entre juillet 2013 et décembre 2018. Ses recherches de terrain, menées en Afrique de l'Ouest et du Centre, s'inscrivent dans le champ du développement et, plus spécifiquement, des questions agricoles et alimentaires, de la lutte contre l'insécurité alimentaire et de la gouvernance des crises alimentaires et nutritionnelles. Entre 2014 et 2017, il a coordonné un projet de recherche partenarial public-privé (université Paris 1/Groupe Nutriset) consacré à la sécurisation et la satisfaction alimentaires avec des jeunes chercheurs et chercheuses. Il est corédacteur en chef de la Revue internationale des études du développement depuis juin 2015.

\footnotetext{
${ }^{25}$ Particulièrement visible depuis l'élaboration d'un cadre harmonisé d'analyse et de traitement des crises alimentaires et nutritionnelles (Integrated Food Security and Humanitarian Phase Classification), élaboré conjointement par la FAO et la PAM dès 2006, et régulièrement amélioré depuis (Partenaires globaux IPC, 2012).

${ }^{26}$ En contrepartie, il convient aussi de rappeler les contraintes auxquelles ils font souvent face : accès difficile au crédit bancaire ; quantité et qualité incertaine lors de la collecte de denrées locales, vétusté des infrastructures de transport, multiplicité des contrôles policiers et des taxations abusives (Pourcet, 1986 ; Egg et Herrera, 1998).
} 


\section{Références bibliographiques}

Abo-El-Wafa Hany, Yeshitela Kumelachew and Pauleit Stephan, 2017. "Exploring the future of rural-urban connections in sub-Saharan Africa: modelling urban expansion and its impact on food production in the Addis Ababa region ", Geografisk Tidsskrift-Danish Journal of Geography, vol. 117, n² 2, p. 68-81.

Acloque Desmulier Delphine, Gasselin Pierre, Rouillé d'Orfeuil Henri (coord.), 2014. Agricultures familiales: trajectoires, modernités et controverses, Revue Tiers-Monde, Paris, Armand Colin, $\mathrm{n}^{\circ} 220,262 \mathrm{p}$.

AFD, 2015. Les profondes mutations des consommations alimentaires en Afrique, Question de développement, Synthèses des études et recherches de l'AFD, juin 2015, 4 p.

Allen Patricia and Sachs Carolyn, 2009. "Women and food chains : The gendered politics of food ", International Journal of Sociology of Food and Agriculture, vol. 15, n 1, 23 p.

Amanor Kojo Sebastian, 2009. "Global Food Chains, African Smallholders and World Bank Governance », Journal of Agrarian Change, vol. 9, $\mathrm{n}^{\circ}$ 2, p. 247-262.

Araujo Bonjean Catherine, Brunelin Stéphanie et Simonet Catherine, 2010. Prévenir les crises alimentaires au Sahel : des indicateurs basés sur les prix de marché, Document de travail, $n^{\circ}$ 95, AFD, Paris, 134 p.

Arditi Claude, Janin Pierre et Marie Alain, (éds.), 2011. La lutte contre l'insécurité alimentaire au Mali : réalités et faux semblants, Paris, Karthala, Collection Hommes et sociétés, 384 p.

Ariyo Ayodele J., Voh J. P. and Ahmed B., 2001. "Long-term change in food provisioning and marketing in the Kano region, 1960-2000", Drylands Research, Working Paper 34, 66 p.

Azagrande Maurizio, 1997. "Les approches disciplinaires de l'analyse des SADA", Coll. " Aliments dans les villes ", Programme "Approvisionnement et distribution alimentaires des villes", FAO, 55 p.

Armendáriz Vanessa, Armenia Stefano, and Atzori Alberto Stanislao, 2016. « Systemic Analysis of Food Supply and Distribution Systems in City-Region Systems-An Examination of FAO's Policy Guidelines towards Sustainable Agri-Food Systems ", Agriculture, vol. 6, n65, 19 p.

Berton-Ofouémé Yolande, 2017. L'accès à l'alimentation dans les grandes villes (Asie, Afrique, Caraïbes), Paris, L'Harmattan, $272 \mathrm{p}$.

Bertrand Jean-Pierre, Théry Hervé, Waniez Philippe, 1991. « Les Japonais et la mise en valeur des Cerrados au Brésil : pour quelle maîtrise de l'espace et de l'approvisionnement alimentaire ? ", Économie rurale, $\mathrm{n}^{\circ}$ 202203, p. 58-64.

Bilinsky, Paula and Anne Swindale, 2005. Months of Inadequate Household Food Provisioning (MIHFP) for Measurement of Household Food Access: Indicator Guide. Washington D.C.: Food and Nutrition Technical Assistance Project, Academy for Educational Development, $10 \mathrm{p}$.

Blay-Palmer Alison, Santini Guido, Dubbeling Marielle, Renting Henk, Taguchi Makiko and Giordano Thierry, 2018. "Validating the city region food system approach: Enacting inclusive, transformational city region food systems ", Sustainability, 10, 1680, 23 p.

Bognon Sabine, 2014. Les transformations de l'approvisionnement alimentaire dans la métropole parisienne. Trajectoire socio-écologique et construction de proximités. Thèse de doctorat en géographie, Université Paris 1 Panthon-Sorbonne, $413 \mathrm{p}$.

Bonnecase Vincent, 2016. " Le goût du riz. Une valeur sensorielle et politique au Burkina Faso », Genèses, n 104, p. 7-29.

Bourmaud Daniel et D. Darbon Dominique 1990, " La politique du pain : les mots et les choses (Kenya et Zimbabwe) », Politique Africaine, $n^{\circ} 37$, p. 32-44.

Bricas Nicolas, Pichot Jean-Pascal et Wack A.-L. (coord.), 2004. "L'alimentation des villes », Cahiers agricultures, vol. $13, \mathrm{n}^{\circ} 1,171 \mathrm{p}$.

Bricas Nicolas, Tchamda Claude, Martin Pauline, 2016. « Les villes d’Afrique de l'Ouest et du Centre sont-elles si dépendantes des importations alimentaires ? ", Cahiers Agricultures, vol. 25, 10 p.

Calas Bernard, 1999. "Les paradoxes des rapports villes-campagnes à travers l'analyse du ravitaillement kampalais », p. 88-103, in : Chaléard Jean-Louis, Dubresson Alain (éds), 1999. Villes et campagnes dans les pays du Sud. Géographie des relations, Editions Karthala.

Chaléard Jean-Louis, Moustier Paule et Leplaideur Alain, 2002. « L'approvisionnement vivrier des villes en Guinée : entre fragilité et dynamisme ", Autrepart, n²3, p. 5-23. 
Chaumet Jean-Marc et Pouch Thierry, 2017. La Chine au risque de la dépendance alimentaire, Presses universitaires de Rennes, $211 \mathrm{p}$.

Chaumet Jean-Marc, 2015. « Nourrir la Chine : géopolitique d’un défi alimentaire », Hérodote, vol. 156, n. 1, p. 7392.

Chevalier Sophie, 2015. "Food, malls and the politics of consumption: South Africa's new middle class", Development Southern Africa, vol. 32, $\mathrm{n}^{\circ}$ 1, p. 118-129.

Commission sur l'avenir de l'agriculture et de l'agroalimentaire du Québec (CAAAQ), 2008. Agriculture et agroalimentaire : assurer et bâtir l'avenir. Rapport final, $272 \mathrm{p}$.

Cossi Hinnou Léonard, Mongbo Roch L., Sanyang Sidi, Raboanarielina Cara M. et Kamanda Josey, 2016. « Plateforme d'innovation et gouvernance des chaînes de valeur du riz local au Bénin : entre jeux de pouvoir et démocratie interne ", 10es Journées de Recherches en Sciences Sociales, Paris - la Défense, 8-9 décembre 2016, 32 p.

Coussy Jean, 1990. "Politiques agro-alimentaires et programmes d'ajustement structurel en Afrique subsaharienne ", p. 13-37, in : Blanc-Pamard Chantal, Bonefond Philippe, Couty Philippe, Lassailly-Jacob Véronique, Marchal Jean-Yves et Piton Florence (eds.), 1990. Dynamiques des systèmes agraires : la dimension économique, Paris, ORSTOM, Coll. Colloques et séminaires.

Chiffoleau Yuna, 2008. "Les circuits courts de commercialisation en agriculture : diversité et enjeux pour le développement durable », p. 21-30, in : Maréchal G. (coord.), 2008. Les circuits couts alimentaires. Bien manger dans les territoires, Dijon, Educagri éditions.

Crush Jonathan and Frayne Bruce, 2011. "Supermarket expansion and the Informal food economy in southern african cities: Implications for urban food security ", Journal of Southern African Studies, vol. 37, $n^{\circ} 4, p^{\text {p. }} 781$ 807.

Delisle Hélène, 1998. "La sécurité alimentaire, ses liens avec la nutrition et la santé ", Canadian Journal of Development Studies / Revue canadienne d'études du développement, vol. 19, n 4, p. 307-329.

Deverre Christian et Lamine Claire, 2010. «Les systèmes agroalimentaires alternatifs. Une revue de travaux anglophones en sciences sociales ", Économie Rurale, $\mathrm{n}^{\circ} 317$, p. 57-73.

Elbehri Aziz (ed.), 2013. Rebuilding West Africa's food potential: Policies and market incentives for smallholderinclusive food value chains, FAO: Rome, Italy, $593 \mathrm{p}$.

Egg Johny et Herrera Javier (coord.), 1998. Echanges transfrontaliers et intégration régionale, Autrepart, $\mathrm{n}^{\circ}$ 6, IRD, $210 \mathrm{p}$.

Engels Bettina, 2015. "Contentious Politics of Scale: The Global Food Price Crisis and Local Protest in Burkina Faso", Social Movement Studies: Journal of Social, Cultural and Political Protest, vol. 14, n² 2, p. 180-194.

Enten François, 2017. «Temporalités des systèmes d'alerte précoce et extraversion de l'État éthiopien (20032016) ", Politique africaine, $n^{\circ} 145$, p. 65-83.

Frayne Bruce, 2010. "Pathways of food: mobility and food transfers in Southern Africa cities", International Development Planning Review, vol. 32, n³-4, p. 291-310.

Festas Laurent, 2006. Enjeux et dynamiques du commerce de produits alimentaires à Bobo-Dioulasso (Burkina Faso) et Tamale (Ghana), Université de Paris-Nanterre, France, 407 p.

Franqueville André, 1997. "Les espaces géographiques du ravitaillement urbain. Cadre conceptuel ", Programme FAO Approvisionnement et distribution alimentaires des villes, Revue Aliments dans les villes, vol. 1, 27 p.

Frayne Bruce, 2010. "Pathways of food: mobility and foodtransfers in Southern African cities", International Development Planning Review, vol. 32, n³-4, p. 291-310.

Fromageot Audrey, 2005. "Dépasser l'enclavement: le maraîchage des savanes et l'approvisionnement d'Abidjan », Espace, populations, sociétés, p. 83-98.

Fofiri Nzossie Eric-Joël, 2013. Les déterminants de l'offre alimentaire vivrière dans les villes du Nord du Cameroun, Thèse de Doctorat de géographie économique, Université de Ngaoundéré, Cameroun, $441 \mathrm{p}$.

Galtier Franck et Vindel Bruno (collab.), 2012. Gérer l'instabilité des prix alimentaires dans les pays en développement Une analyse critique des stratégies et des instruments, AFD, Paris, décembre 2012, col. À savoir, $312 \mathrm{p}$.

Goossens Frank, Minten Bart, Tollens Eric, 1994. Nourrir Kinshasa. L'approvisionnement local d'une métropole africaine, Paris, L'Harmattan, $397 \mathrm{p}$. 
Grégoire Emmanuel, Kobiané Jean-François et Lange Marie-France, 2018. L'État réhabilité en Afrique. Réinventer les politiques publiques à l'ère néolibérale, Paris, Karthala, 355 p.

Guyer Jane I. (ed.), 1987, Feeding African Cities: Studies in Regional Social History, Manchester, Manchester University Press.

Hatcheu Tchawe Emil, 2003. L'approvisionnement et la distribution alimentaires à Douala (Cameroun) : logiques sociales et pratiques spatiales des acteurs, thèse de doctorat unique, Université de Paris I Panthéon Sorbonne, $455 \mathrm{p}$.

Hattemer C. et Sierra Alexis, 2012. "L'approvisionnement alimentaire de Lima au défi de la planification stratégique ", $13^{\text {ème }}$ Conférence N-Aerus, La ville inégalitaire, espace contestés, gouvernance en tension, 22 - 24 novembre, Paris, $17 \mathrm{p}$.

Héron R., 2016, Les mangeurs urbains burkinabè, entre satisfaction et sécurisation alimentaires, Thèse de doctorat en socio-anthropologie, Université de Paris 1 Panthéon-Sorbonne, $435 \mathrm{p}$.

Hinrichs C. Clare, 2003. "The practise and politics of food system localization », Journal of Rural Studies, vol. 19, p. 33-45.

Hinrichs C. Clare, 2013. « Regionalizing food security? Imperatives, intersections and contestations in a post-9/11 world ", Journal of Rural Studies, vol. 29, p. 7-18.

Hossain Naomi and Kalita Devangana, 2014. "Moral economy in a global era: the politics of provisions during contemporary food price spikes », The Journal of Peasant Studies, vol. 41, n5, p. 815-831.

Hubert Bernard, 2006. "Introduction. Sécuriser l'alimentation de la planète ", p. 11-22, in : Hubert Bernard, Clément Olivier (éds.), 2006. Le monde peut-il nourrit tout le monde ? Sécuriser l'alimentation de la planète. IRDEditions Quae, $161 \mathrm{p}$.

Hugon Philippe, 1997. "Pluralité des filières d'approvisionnement alimentaire dans les villes d'Afrique francophone ", Aliments dans les villes, FAO, Rome, 35 p.

Janin Pierre, 2008. "Le soleil des indépendances (alimentaires) " ou la mise en scène de la lutte contre la faim au Mali et au Sénégal », p. 92-117, in : Janin Pierre et Giblin Béatrice, 2008. (éd.), Les enjeux de la crise alimentaire mondiale, Paris, La Découverte, Hérodote, $\mathrm{n}^{\circ} 131,206 \mathrm{p}$.

Janin Pierre, 2018. "Les politiques alimentaires en Afrique de l'Ouest : réponse au risque ou facteur d'insécurité ? ", p. 165-188, in : Grégoire Emmanuel, Kobiané Jean-François et Lange Marie-France (éd.), 2018. L'État réhabilité en Afrique. Réinventer les politiques publiques à l'ère néolibérale, Coll. Hommes et sociétés, Karthala, Paris.

Karg Hanna, Drechsel Pay, Akoto-Danso Edmund K., Glaser Rüdiger, Nyarko George and Buerkert Andreas, 2016. "Foodsheds and city region food systems in two West African cities ? Sustainability vol. 8, $\mathrm{n}^{\circ} 12,32 \mathrm{p}$.

Kloppenburg Jack, Hendrickson John and Stevenson G. W., 1996. "Coming in to the foodshed ", Agricultural and Human Values, vol. 13, $n^{\circ} 3$, p. 33-42.

Labonne Michel, 1986. "L'autosuffisance alimentaire en question », p. 357-367, in : Courade Georges et al., (éd.), 1986. Nourrir les villes en Afrique Subsaharienne, L'Harmattan, $421 \mathrm{p}$.

Lavers Tom, 2013. « Food security and social protection in highland Ethiopia: Linking the Productive Safety Net to the land question ", Journal of Modern African Studies, vol. 51, n³, p. 459-485.

Luo Jianli, Ji Chen, Qiu Chunxiao and Jia Fu, 2018. « Agri-food supply chain management: Bibliometric and content analyses ", Sustainability, 10, 1573, 22 p.

Lutringer Christine, 2018. « Le ciblage des politiques agricoles en Inde : les implications pour les paysans du système d'approvisionnement public ", in : Ancey Véronique, Chevalier Pascal, Sourisseau Jean-Michel (coord.), 2018. Catégorisations politiques et transformations sociales en agriculture, Cahiers agricultures, vol. 26, nº 4 , $\mathrm{p}$.

Hrabanski Marie, 2011. "Souveraineté alimentaire. Mobilisations collectives agricoles et instrumentalisations multiples d'un concept transnational », Revue Tiers Monde, n²07, p. 151-168.

Maestre Mar, Poole Nigel, Henson Spencer, 2017. « Assessing food value chain pathways, linkages and impacts for better nutrition of vulnerable groups ", Food Policy, vol. 68, p. 31-39.

Mander Harsh, 2015. State food provisioning as social protection. Debating India's national food security law. Right to food study, FAO, $80 \mathrm{p}$.

Marsden Terry, Banks Jo and Bristow Gillian, 2000. "Food supply chain approaches: Exploring their role in rural development ", Sociologia Ruralis, vol. 40, n 4, p. 425-438. 
Mormont Claude, 2007. "Pourquoi un programme centré sur la souveraineté alimentaire ", in: Charlier Sophie, Warnotte Gérard (coord.), 2007. La souveraineté alimentaire: Regards croisés, Presses Universitaires de Louvain, $330 \mathrm{p}$.

Moruzzi Marques Paulo Eduardo et Le Moal Marcos Freitas, 2015. « Le Programme d’Acquisition d'Aliments (PAA) au Brésil : l'agriculture locale et familiale au cœur de l'action publique en vue de la sécurité alimentaire", VertigO - la revue électronique en sciences de l'environnement, vol. 14, $\mathrm{n}^{\circ} 1$.

Mottet Eric, 2016. " Autosuffisance et sécurisation alimentaire chinoise : quelle stratégie régionale ? ", Diplomatie, $n^{\circ} 79$, p. 87-91.

Moustier Paule, 1999. « Complémentarité entre agriculture urbaine et agriculture rurale », p. 41-55, in : Olanrewaju B. Smith (dir.), 1999. Agriculture urbaine en Afrique de l'Ouest. Contribution à la sécurité alimentaire et à l'assainissement des villes, Centre de recherches pour le développement international, Ottawa (Ontario), Canada/Centre technique de coopération agricole et rurale ACP-UE, Wagenigen, Pays-Bas.

Moustier Paule and Renting H., 2015. "Urban agriculture and short chain food marketing in developing countries », p. 121-138, in : de Zeeuw, H., Drechsel, P., (eds.), 2015. Cities and agriculture. Developing resilient urban food systems, Routledge: London, UK.

Nally David, 2011. "The biopolitics of food provisioning ", Transactions of the Institute of British Geographers, vol. $36, n^{\circ} 1$, p. 37-53.

OCDE, 2013. Peuplement, marché et sécurité alimentaire, Cahiers de l'Afrique de l'Ouest, OCDE, $208 \mathrm{p}$.

Ostrom Marcia Ruth, 2009. «Community Supported Agriculture as an Agent of Change”, p. 99-120, in: Hinrichs C.C. et Lyson T.A. (éds.), 2009. Remaking the North American Food System: Strategies for sustainability, University of Nebraska Press, $384 \mathrm{p}$.

Padilla Martine, 1997. "La sécurité alimentaire des villes africaines: le rôle des SADA", Collection "Aliments des villes", programme Approvionnement et distribution alimentaires des villes", FAO, $46 \mathrm{p}$.

Padilla Martine, Bencharif A. 2001. «Approvisionnement alimentaire des villes : concepts et méthodes d'analyse des filières et marchés », p. 262-279, in : Padilla M., Ben Saïd T., Hassainya J., Le Grusse P. (éds.). Les filières et marchés du lait et dérivés en Méditerranée : état des lieux, problématique et méthodologie pour la recherche, Montpellier, CIHEAM, Options Méditerranéennes, Série B. Etudes et Recherches, $n^{\circ} 32$.

PAM, 2013. Gestion de la chaîne d'approvisionnement des aliments nutritifs spécialisés, Rome, 260 p.

Parfitt, Julian, Mark Barthel, and Sarah Macnaughton. 2010. “Food Waste within Food Supply Chains: Quantification and Potential for Change to 2050", Philosophical Transactions of the Royal Society B, n 365, p. 3065-3081.

Partenaires globaux IPC, 2012. Cadre intégré de classification de la sécurité alimentaire : Manuel technique version 2.0. Preuves et normes pour une meilleure prise de décision en sécurité alimentaire, FAO, Rome, $152 \mathrm{p}$.

Petrini Carlo, 2006. Slow food. Bon, propre et juste. Ethique de la gastronomie et souveraineté alimentaire, Torrent, Editions Yves Michel, 328 p.

Porter Gina, Lyon Fergus, Potts Deborah, 2007. « Market institutions and urban food supply in West and Southern Africa: a review », Progress in Development Studies, vol. 7, n², p. 115-34.

Pouch Thierry, 2009. "Malaise dans l'approvisionnement alimentaire du monde ", Géoéconomie, vol. 50, n 3, p. 117-123.

Pouch Thierry, 2011. "La résurgence de la souveraineté alimentaire ", Economies et sociétés, Série Systèmes agroalimentaires, AG, $n^{\circ} 33$, p. 1719-1735.

Poussart-Vanier Marie, 2006. Jeux d'acteurs dans le système alimentaire burkinabé. Normes, conflits et compromis dans le marché céréalier et la gestion de l'aide alimentaire, Thèse en sociologie, IEDES-Université Paris 1, 284 p.

Pourcet Guy, 1986. "Le système d'approvisionnement d'Antanarivo en crise ", p. 215-230, in : Bricas N.,Courade G., Coussy J., Hugon Ph. et Muchnick J., 1986. Nourrir les villes en Afrique subsaharienne, Col. Villes et entreprises, L'Harmattan, $421 \mathrm{p}$.

Rapoport Hillel, 1993. "L'approvisionnement vivrier de Kinshasa (Zaïre): stratégies d'adaptation à la crise du système alimentaire ", Cahiers de sciences humaines, ORSTOM, vol. 29, n 4, p. 695-711.

Rastoin Jean-Louis, 2015. « La sécurité alimentaire en Méditerranée :

Rastoin Jean-Louis et Ghersi Gérard, 2010. Le système alimentaire mondial. Concepts e tméthodes, analyses et dynamiques, Versailles, Quae, $584 \mathrm{p}$. 
Reinert Kenneth A., 2015. " Food security as basic goods provision », World Medical \& Health Policy, vol. 7, n 3, p. 171-186.

Rittel Horst W. H. and Webber M. M., 1973. « Dilemmas in a general theory of Planning », Policy Science, vol. 4, p. 155-169.

Robineau Ophélie, 2013. Vivre de l'agriculture dans la ville africaine. Géographie des arrangements entre acteurs à Bobo-Dioulasso, Burkina Faso. PhD thesis, Université Montpellier 3, CIRAD, INRA. 352 p.

Robineau Ophélie et Soulard Christophe-Toussaint, 2017. "Understanding the complexity of agricultural-urban relationships through a systemic approach. The case of Bobo-Dioulasso, West Africa », Dossier L'agriculture dans le système alimentaire urbain : continuités et innovations ", Nature Sciences Sociétés, vol. 25, n 1, p. 36-47.

Siobhan Kelly and Swensson Luana F.J., 2017. Leveraging institutional food procurement for linking small farmers to markets: Findings from WFP's Purchase for Progress initiative and Brazil's food procurement programmes, FAO Agricultural Development Economics Technical Study 1. Rome, FAO. 101 p.

Tawodzera Godfrey, 2013. "Rural-Urban Transfers and Household Food Security in Harare's Crisis Context ", Journal of Food \& Nutritional Disorder, vol. 2, $n^{\circ} 5,10 \mathrm{p}$.

Temple Ludovic, Ngassam Sylvain et Nkamleu Guy, 2009. « Filières d'approvisionnement en ignames de Douala et changements technologiques " Economies et sociétés, développement, croissance et progrès, Presses de I'ISMEA, Paris, AG, n 31, p. 1899-1912.

Touzard Jean-Marc, Temple Ludovic, 2012. " Sécurisation alimentaire et innovations dans l'agriculture et l'agroalimentaire : vers un nouvel agenda de recherche ? Une revue de la littérature ", Cahiers Agricultures, vol. 21, p. 293-301.

Van der Ploeg J. D., Ye J. and Schneider S., 2012. " Rural development through the construction of new, nested, markets: comparative perspectives from China, Brazil and the European Union ", Journal of Peasant Studies, vol. 39, $n^{\circ} 1$, p. 133-173.

Wade Idrissa et Lançon Frédéric, 2015. « Urbanisation, changements des habitudes alimentaires et transformations rurales en afrique de l'Ouest ", Global Development Network Conference 2015 Agriculture for Sustainable Growth: Challenges and Opportunities for a New "Green Revolution », 8 p.

Watts D.C.H., Ibery B. and Maye D., 2005. « Making reconnections in agro-food geography: alternative systems of food provision », Progress in Human Geography, vol. 29, n 1,p. 22-40. 